\title{
Multicenter Validation of Histopathologic Tumor Regression Grade After Neoadjuvant Chemotherapy in Muscle-invasive Bladder Carcinoma
}

\author{
Charlotte S. Voskuilen, MD, * Htoo Zarni Oo, MD, PhD, $\dagger$ Vera Genitsch, MD, $\neq$ \\ Laura A. Smit, MD, PhD, \& Alvaro Vidal, MD, \| Manuel Meneses, MD, \| Andrea Necchi, MD, ๆ \\ Maurizio Colecchia, MD,\# Evanguelos Xylinas, MD,** Jacqueline Fontugne, MD, †† \\ Mathilde Sibony, MD, PhD, †† Morgan Rouprêt, MD, PhD, $\neq \neq$ Louis Lenfant, MD, $\neq \neq$ \\ Jean-François Côté, MD, PhD, §£ Lorenz Buser, MD, || Karim Saba, MD, $\uparrow \uparrow \mid$ \\ Marc A. Furrer, MD,\#\# Michiel S. van der Heijden, MD, PhD, *** Mads Daugaard, PhD, $\dagger$ \\ Peter C. Black, MD, † Bas W.G. van Rhijn, MD, PhD, ${ }^{*}$ Kees Hendricksen, MD, PhD, * \\ Cédric Poyet, MD, $1 \uparrow$ and Roland Seiler, MD\#\#
}

\begin{abstract}
Response classification after neoadjuvant chemotherapy in muscle-invasive bladder carcinoma is based on the TNM stage at radical cystectomy. We recently showed that histopathologic tumor regression grades (TRGs) add prognostic information to TNM. Our aim was to validate the prognostic significance of TRG in muscle-invasive bladder cancer in a multicenter setting. We enrolled 389 patients who underwent cisplatin-based chemotherapy before radical cystectomy in 8 centers between 2010 and 2016. Median follow-up was 2.2 years. TRG was determined in radical cystectomy specimens by local pathologists. Central pathology review was conducted in $20 \%$ of cases, which were randomly selected. The
\end{abstract}

From the Departments of *Urology; §Pathology; *** Medical Oncology, The Netherlands Cancer Institute-Antoni van Leeuwenhoek Hospital, Amsterdam, The Netherlands; †Department of Urologic Sciences, Vancouver Prostate Centre, University of British Columbia, Vancouver, BC, Canada; \$Institute of Pathology; \#\#Department of Urology, University of Bern, Bern; \|||Institute of Pathology and Molecular Pathology, University Hospital Zurich; qףDepartment of Urology, University Hospital Zürich, University of Zürich, Zürich, Switzerland; \|Faculty of Medicine, Instituto Oncológico FALP, Santiago, Chile; Departments of $\uparrow$ Medical Oncology; \#Pathology, Fondazione IRCCS Istituto Nazionale dei Tumori, Milan, Italy; **Department of Urology, Hôpital Cochin, Paris Descartes University; ††Department of Pathology, Institut Curie; Departments of \#Urology; and §§Pathology, Sorbonne Université, GRC n5, ONCOTYPE-URO, AP-HP, Hôpital Pitié-Salpêtrière, Paris, France.

C.S.V. and H.Z.O. shared first authorship.

C.P. and R.S. shared senior authorship.

Conflicts of Interest and Source of Funding: The authors have disclosed that they have no significant relationships with, or financial interest in, any commercial companies pertaining to this article.

Correspondence: Charlotte S. Voskuilen, MD, Department of Urology, The Netherlands Cancer Institute-Antoni van Leeuwenhoek Hospital, Plesmanlaan 121 Amsterdam 1066 CX, The Netherlands (e-mail: c.voskuilen@nki.nl).

Supplemental Digital Content is available for this article. Direct URL citations appear in the printed text and are provided in the HTML and PDF versions of this article on the journal's website, www.ajsp. com.

Copyright (C) 2019 Wolters Kluwer Health, Inc. All rights reserved. major response was defined as $\leq \mathrm{pT} 1 \mathrm{~N} 0$. The remaining patients were grouped into partial responders ( $\geq$ ypT2N0-3 and TRG 2) and nonresponders ( $\geq$ ypT2N0-3 and TRG 3). TRG was successfully determined in all cases, and interobserver agreement in central pathology review was high $(\kappa=0.83)$. After combining TRG and TNM, $47 \%, 15 \%$, and $38 \%$ of patients were major, partial, and nonresponders, respectively. Combination of TRG and TNM showed significant prognostic discrimination of overall survival (major responder: reference; partial responder: hazard ratio 3.5 [95\% confidence interval: 1.8-6.8]; nonresponder: hazard ratio 6.1 [95\% confidence interval: 3.6-10.3]). This discrimination was superior compared with TNM staging alone, supported by 2 goodnessof-fit criteria $(P=0.041)$. TRG is a simple, reproducible histopathologic measurement of response to neoadjuvant chemotherapy in muscle-invasive bladder cancer. Integrating TRG with TNM staging resulted in significantly better prognostic stratification than TNM staging alone.

Key Words: bladder carcinoma, neoadjuvant chemotherapy, tumor regression grade, urothelial carcinoma

(Am J Surg Pathol 2019;43:1600-1610)

$\mathrm{N}$ eoadjuvant chemotherapy followed by radical cystectomy is the gold-standard treatment for patients with muscleinvasive bladder carcinoma. ${ }^{1}$ However, up to $60 \%$ of patients treated with neoadjuvant chemotherapy have residual muscleinvasive bladder cancer at radical cystectomy. ${ }^{2}$ These patients have a significantly worse overall survival (OS) compared with patients achieving a pathologic complete response after neoadjuvant chemotherapy. 3,4

As patients who do not respond to neoadjuvant chemotherapy have no obvious benefit from therapy but suffer potential toxicity of therapy and delay in definitive surgery, it stands to reason that the ability to select patients for neoadjuvant chemotherapy based on the likelihood of response would improve patient outcomes. Therefore, research has 
focused on discovery and validation of predictive biomarkers. However, previous studies investigating predictive biomarkers have used different TNM categories to define response to neoadjuvant chemotherapy. ${ }^{5-7}$ This variability may indicate the need for a more granular definition of response, determined in bladder carcinoma treated with neoadjuvant chemotherapy. Furthermore, while it is presumed that patients with residual muscle-invasive bladder cancer after neoadjuvant chemotherapy have derived no benefit from neoadjuvant chemotherapy, this has not been definitively demonstrated.

Histopathologic tumor regression grades (TRGs), which quantify the extent of tumor response to systemic treatment, have shown to be a prognostic factor for patient outcome in several malignancies, including gastric, esophageal, and rectal carcinoma. ${ }^{8-10}$ In muscle-invasive bladder cancer, Fleischmann et $\mathrm{al}^{11}$ found that TRG predicted survival after neoadjuvant chemotherapy independently and better than conventional pathologic TNM response classification. The aim of the present study was to investigate whether the prognostic significance of TRG could be validated in a multicenter cohort of muscle-invasive bladder cancer patients who underwent neoadjuvant chemotherapy followed by radical cystectomy.

\section{MATERIALS AND METHODS}

\section{Patient Population}

We enrolled a consecutive cohort of patients who underwent neoadjuvant chemotherapy followed by radical cystectomy for cT2-4aN0-3M0 bladder carcinoma between 2010 and 2016 in 8 institutions. Although systemic chemotherapy for cN1-3 bladder carcinoma is conventionally referred to as induction chemotherapy, we included it here under the term neoadjuvant chemotherapy. Data were collected in accordance with institutional and national ethical guidelines. Only patients who underwent 3 or 4 cycles of cisplatin-based neoadjuvant chemotherapy were included. Exclusion criteria were a history of pelvic radiotherapy and nonurothelial primary histology. Urothelial carcinoma with squamous and/or glandular differentiation was allowed (Fig. 1). Follow-up was

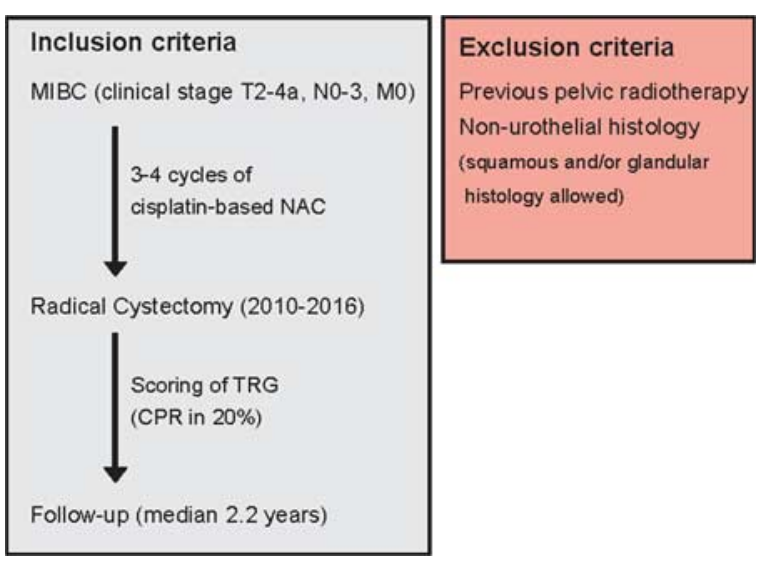

FIGURE 1. Flowchart of patient selection. MIBC indicates muscleinvasive bladder cancer; NAC, neoadjuvant chemotherapy. performed according to institutional protocols. Generally, patients were evaluated every 3 to 4 months for the first year after radical cystectomy, semiannually for the second year, and annually thereafter.

\section{Pathology}

Each center processed the radical cystectomy specimens according to its own institutional protocols. In general, protocols for fixation and pathologic examination were similar between pathology departments. Radical cystectomy specimens were fixed in $10 \%$ neutral buffered formalin ( $4 \%$ formaldehyde) for 24 to 48 hours at room temperature. Characteristics of bladder lesions (residual tumors, ulcers, scars) were described (location, relation to the bladder wall and surrounding tissues), and corresponding tissue samples were taken for histologic examination. Generally, samples from the bladder neck with trigone, dome, and anterior and posterior wall, and the resection margins of ureters and urethra were embedded. Microscopically, tumor grade and tumor stage were noted. Lymph node (LN) specimens were examined by inspection and palpation, and all macroscopically detected LNs were embedded completely. For this study, all hematoxylin \& eosin-stained sections from all patients were re-evaluated by uropathologists (H.Z.O., V.G., L.A.S., J.F., J.F.C., M.M., M.C., and L.B.) in each center. Scoring of regression is described in detail in the next paragraph.

A random selection of $20 \%$ of cases from each center was reviewed independently by 2 pathologists: the institution's local uropathologist and a central pathology reviewer (V.G.). One center (INT Milan) could not participate in central pathology review (CPR) due to logistical constraints. All pathologists were blinded from clinical information, treatment regimens, and study endpoints. In addition, the central pathology reviewer was blinded from other pathologists' interpretations. Major pathologic response to neoadjuvant chemotherapy according to conventional TNM staging was defined as $\leq y p T 1 N 0$. Nonmajor pathologic response was defined as ypT2-4Nany or ypTanyN1-3.

\section{Classification of TRG}

TRG was based on an estimation of the percentage of viable cancer cells in relation to the macroscopically identifiable tumor bed, indicated by zones of fibrosis in the bladder wall and in the perivesical soft tissue, as previously described. ${ }^{11}$ The following 3 TRGs were distinguished (Fig. 2A, Supplemental Fig. 1, Supplemental Digital Content 1, http://links.lww.com/PAS/A840):

TRG 1: Complete response: the absence of histologically identifiable residual cancer cells and extensive fibrosis of the tumor bed.

TRG 2: Strong response: predominant fibrosis of the tumor bed with residual cancer cells occupying $<50 \%$ of this area.

TRG 3: Weak and no response: residual cancer cells occupying $\geq 50 \%$ of the tumor bed or absence of regressive changes.

This grading system was used separately for primary tumors and LN metastases (Figs. 3, 4). For every patient, 

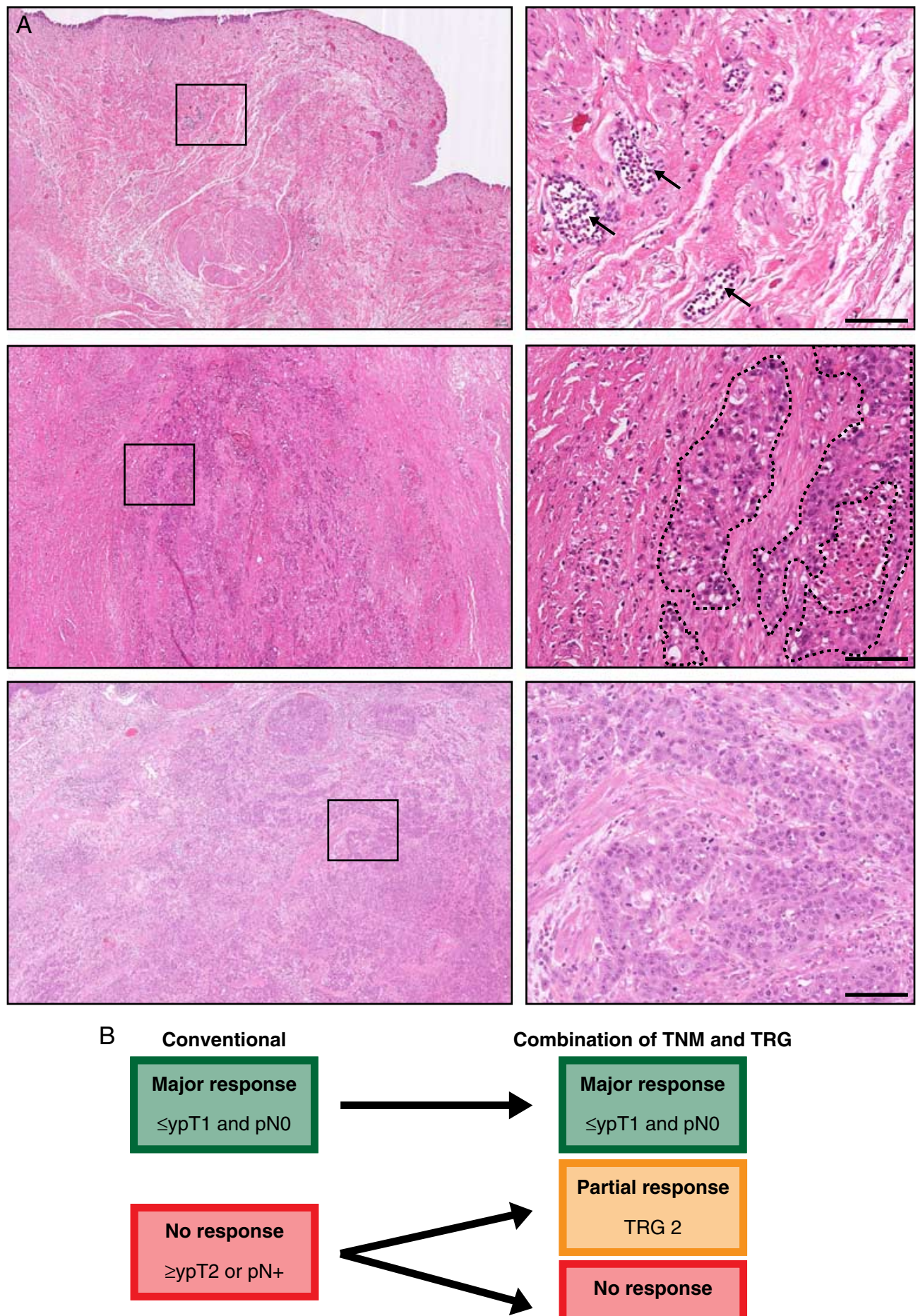

Combination of TNM and TRG

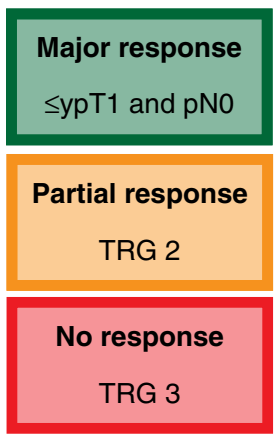

FIGURE 2. A, Representative hematoxylin and eosin images of TRG 1 (uppermost) with inflammatory infiltrate (arrows), TRG 2 (middle), and TRG 3 (lowermost) with muscle-invasive bladder cancer cells, within the original tumor bed. The dashed line delimits cancer cells. The respective zoomed images from the insets (left panel) are shown on the right. Scale bar represents $100 \mu \mathrm{m}$. B, Schematic description of the combination of TRG and TNM stage, showing how TRG stratifies partial and nonresponders on the basis of the histopathologic response.

the dominant TRG, defined as the higher TRG between primary tumor and LNs, was used as the final grade. Response to neoadjuvant chemotherapy was classified into
3 categories on the basis of a combination of TNM stage and TRG: major response, partial response, and no response (Fig. 2B). Major response was defined by the absence of 

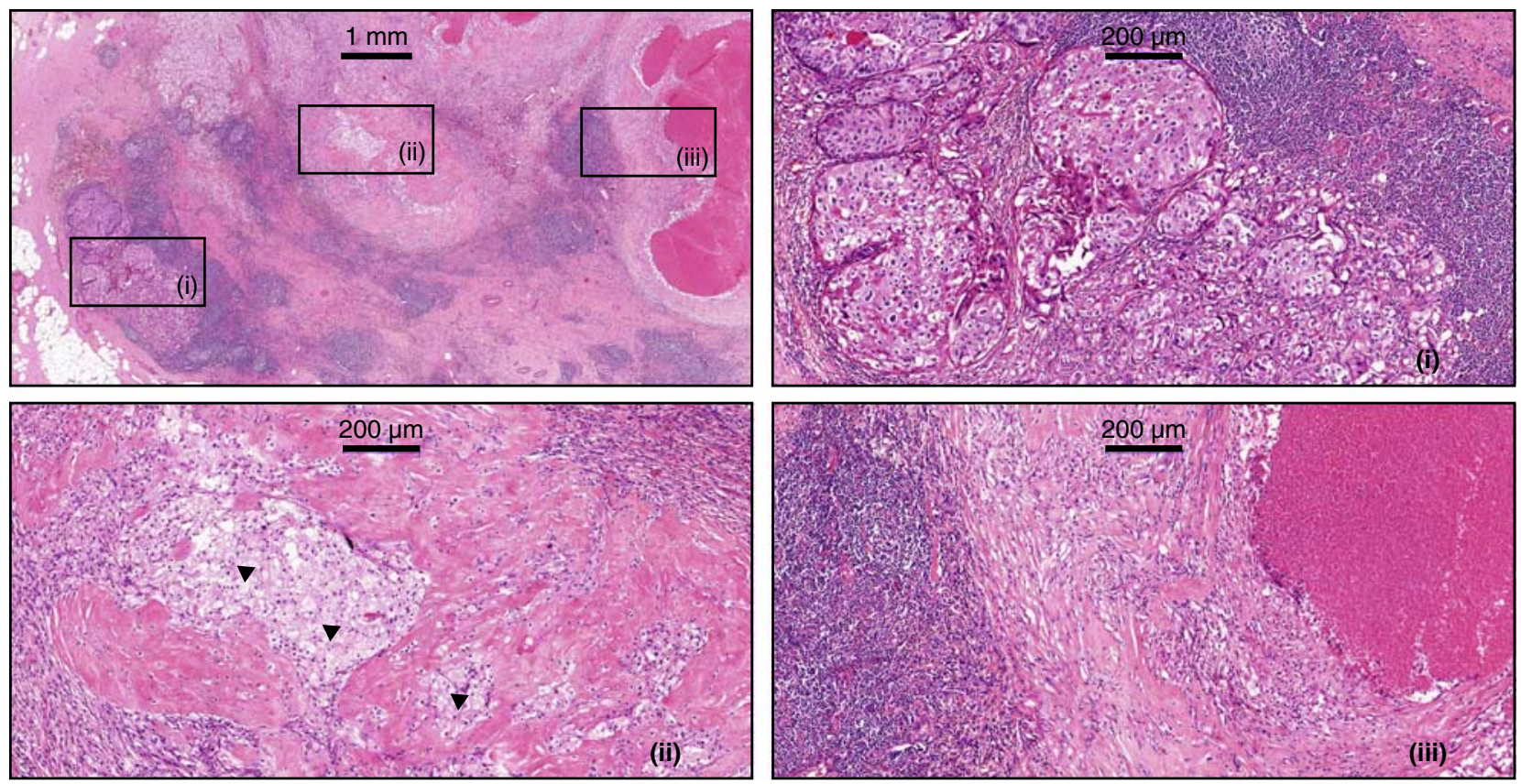

FIGURE 3. Representative hematoxylin and eosin images in a partially regressed LN metastasis with a scanty piece of viable cancer cells, TRG 2. The respective zoomed images (i, ii, iii) of the insets from the original tumor bed (top left, low power view) are shown. (i) Remaining bladder cancer cells in the displaced LN structure. Higher magnification shows dense fibrosis and a nodular accumulation of foamy macrophages (arrowheads) (ii) and nodular zone of necrosis, surrounded by granulation tissue and remaining LN (iii).

muscle-invasive disease and $\mathrm{LN}$ involvement ( $\leq$ ypT1N0). Partial response was defined as $\geq$ ypT2N0-3 with TRG 2 . Finally, patients with $\geq \mathrm{ypT} 2 \mathrm{~N} 0-3$ and TRG 3 were considered to have no response to neoadjuvant chemotherapy.

\section{Statistical Analysis}

Statistical analyses were performed using IBM SPSS Statistics, version 22.0 (IBM Corp., Armonk, NY) and R version 3.4.4 (R Foundation for Statistical Computing, Vienna, Austria). All tests were 2 sided, with the level of significance set at $P$-value $<0.05$. The 3 TRGs were compared with clinicopathologic characteristics using analysis of variance for continuous data and the Pearson $\chi^{2}$ test for categorical data. Kaplan-Meier plots were used to estimate OS from the time of radical cystectomy to the date of death. Comparisons between response groups were made using the log-rank test. Patients still alive were censored at the date of the last follow-up. Median followup was calculated using the reverse Kaplan-Meier method.

The level of agreement between TRG score by the local pathologist and the central pathology reviewer was expressed by means of a Cohen $\kappa$ coefficient. In case of discrepancy between the local pathologist and the central pathology reviewer, the TRG determination of the former was used for further analysis.

The association between pathologic response and OS was assessed using Cox proportional hazard models. To compare response according to the conventional pathologic stage and according to staging including TRG, 2 models were created. Model 1 included age, sex, surgical margin status, and conventional pathologic response as covariates, whereas model 2 included a combination of TNM stage and TRG. Model fit was compared using the likelihood ratio test and the Akaike Information Criterion (AIC). The AIC adjusts the -2 log-likelihood statistic for the number of parameters in the model and number of observations used. A smaller AIC indicates a more desirable model for predicting the outcome.

\section{Sample Size Calculation}

The minimum size of the study cohort was determined by a power analysis based on observed outcomes in the previous study on TRG in muscle-invasive bladder cancer. ${ }^{11}$ The anticipated event rate was $35 \%$. Assuming a Cox regression coefficient of 1.8 and an SD of 0.4, 44 nonresponders were needed to achieve $80 \%$ power at a 0.05 significance level. In addition, $\sim 26$ partial responders were needed $(20 \%$ partial responders in previous data sets). With use of these variables, a total of at least 130 patients was required.

\section{RESULTS}

\section{Patient and Tumor Characteristics}

Radical cystectomy specimens of 389 patients were evaluated. Clinicopathologic characteristics stratified by TRG are shown in Table 1. Lower TRG was associated with lower ypT categories $(P<0.001)$ and with the absence of $\mathrm{LN}$ metastases $(P<0.001)$. There was also a significant association between variant histology (ie, urothelial carcinoma with either squamous differentiation $(\mathrm{n}=34)$ or glandular differentiation $(\mathrm{n}=4))$ and higher TRG, with more patients having variant histology in the TRG 3 group $(P=0.002)$. 

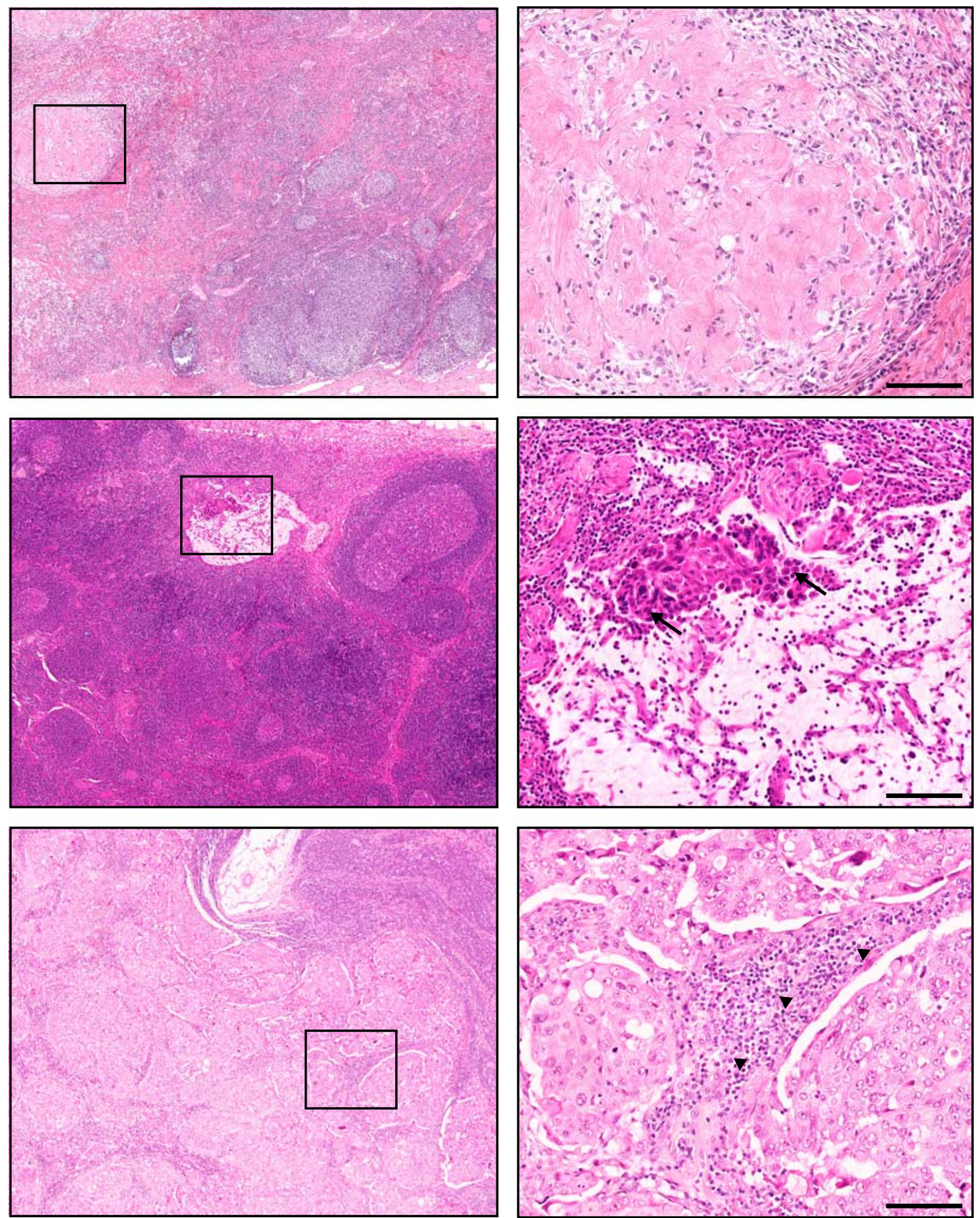

FIGURE 4. Representative hematoxylin and eosin images of LN TRG 1 (uppermost) with fibrotic tumor bed and remaining LN. LN TRG 2 (middle) with necrosis and a small island of residual cancer cells (arrows). LN TRG 3 (lowermost) with cancer cells displacing almost the entire LN and arrowheads show the remaining $L N$. The respective zoomed images from the insets (left panel) are shown on the right. Scale bar represents $100 \mu \mathrm{m}$. 
TABLE 1. Patient and Tumor Characteristics by TRG

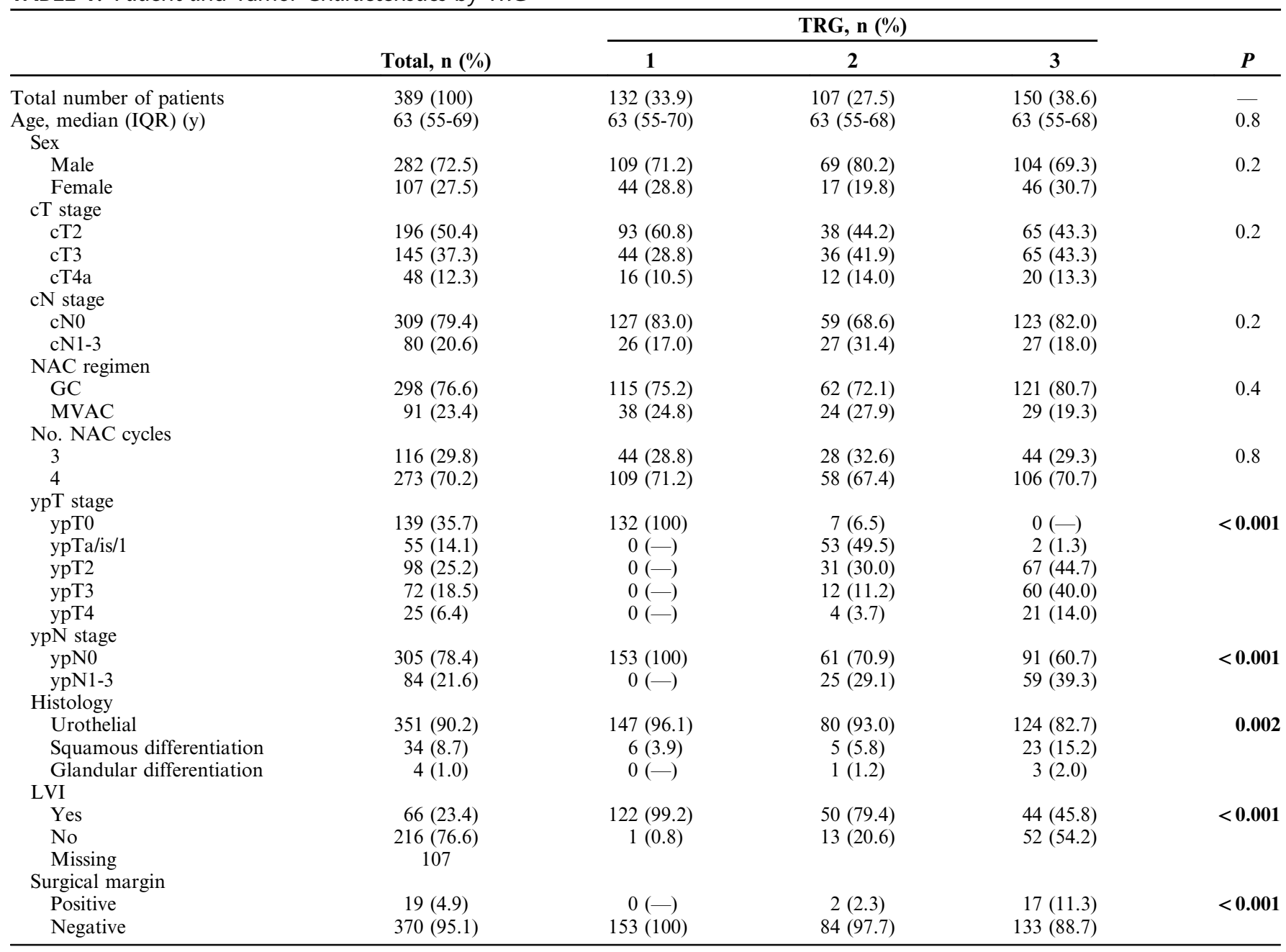

Bold value indicates statistically significant $(P<0.05)$.

GC indicates gemcitabine and cisplatin; IQR, interquartile range; LVI, lymphovascular invasion; MVAC, methotrexate, vinblastine, doxorubicin, and cisplatin; NAC, neoadjuvant chemotherapy.

\section{Pathologic Response}

At the time of radical cystectomy, 139/389 (36\%) patients had no residual primary tumor (ypT0) and 305/389 (78\%) patients had no LN metastases (ypN0). Seven patients had no residual primary tumor but were $\mathrm{LN}$ positive (ypT0N+). According to the conventional TNM response definition, 181/389 patients $(47 \%)$ had a major response, whereas 208/389 (53\%) were nonmajor responders. TRG was successfully determined in all cases. For every patient, the corresponding TRG in the primary tumors and in the LNs is given in Table 2. When

TABLE 2. TRGs of Primary Tumors and LN Metastases

\begin{tabular}{lccc}
\hline & \multicolumn{3}{c}{ Primary Tumor } \\
\cline { 2 - 4 } LN Metastases & TRG 1 & TRG 2 & TRG 3 \\
\hline TRG 1 & 132 & 75 & 91 \\
TRG 2 & 7 & 25 & 0 \\
TRG 3 & 0 & 0 & 59 \\
\hline
\end{tabular}

combining TRG with the TNM stages, $181(47 \%), 59(15 \%)$, and $149(38 \%)$ patients were major, partial, and nonresponders, respectively.

\section{Central Pathology Review}

Local pathologists and the central pathology reviewer agreed on TRG in $88 \%(46 / 52)$ of cases. The overall kappa between the central pathology reviewer and local pathologists was 0.82 .

\section{Survival}

During a median follow-up of 2.2 years $(95 \%$ confidence interval [CI]: 2.0-2.4), 112/389 patients had a cancer recurrence, and 98/389 patients died. In the TRG 1 group, median OS was not reached, whereas, in the TRG 2 and TRG 3 groups, median OS was 3.3 (95\% CI: $2.4-4.1)$ and 2.6 years $(95 \%$ CI: 1.6-3.6), respectively $(P=0.026)$. As expected, higher ypT and higher ypN stage were significantly associated with worse OS in Kaplan-Meier analysis $(P<0.001$, Figs. 5A, B). Positive 

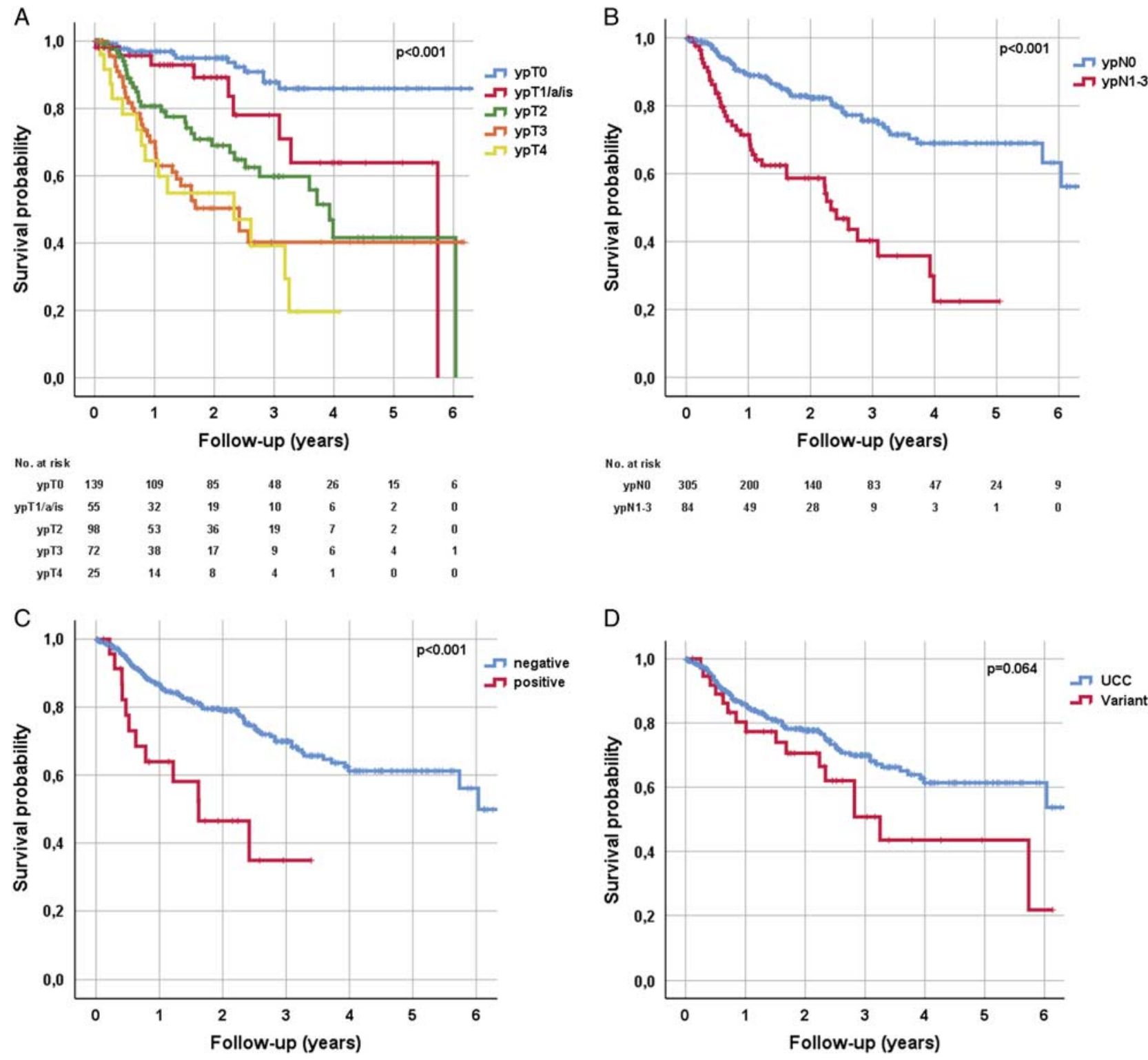

D
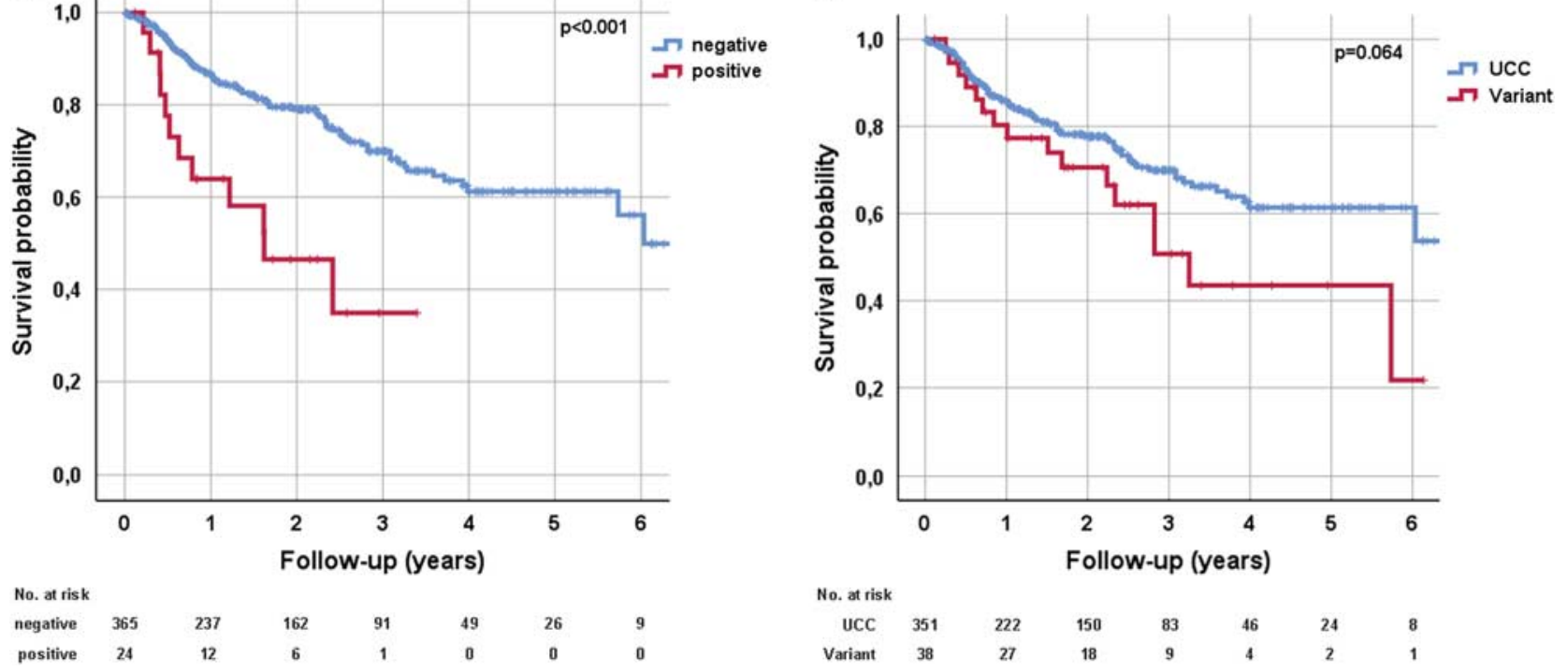

FIGURE 5. Kaplan-Meier plots for OS stratified according to ypT stage (A), ypN stage (B), surgical margin status (C), and histology type (D). UCC indicates urothelial carcinoma.

surgical margin status was also significantly associated with worse OS $(P<0.001$, Fig. 5C), whereas variant histology was not $(P=0.06$, Fig. 5D).

Kaplan-Meier analysis showed a significant difference in OS between major responders and nonmajor responders on the basis of TNM staging alone $(P<0.001$, Fig. 6A). Integrating TRG in the conventional TNM staging showed further stratification of survival curves, discriminating 3 groups of patients with significantly different OS $(P<0.001$, Fig. 6B). Subgroup analysis of TRG 2 versus TRG 3 within ypT2 or ypT3/4 stages showed no significant difference in OS (ypT2: TRG 2 [n=31] vs. TRG $3[\mathrm{n}=67], P=0.3$; ypT3/4:
TRG $2[\mathrm{n}=16]$ vs. TRG $3[\mathrm{n}=81], P=0.2$, Figs. 7A, B). Subgroup analysis of TRG 2 versus TRG 3 among patients with $\mathrm{LN}$ metastases $(\mathrm{n}=84)$ showed a significant difference in $\mathrm{OS}(P=0.03$, Fig. $7 \mathrm{C})$.

In univariable analysis, a higher TRG significantly correlated with worse survival $(P<0.001$, Table 3$)$. Other factors associated with worse OS were higher ypT and ypN stages and positive surgical margins (all $P<0.001$ ). In multivariable analysis, a combination of TRG and TNM showed a significant association with survival $(P<0.001)$, discriminating 3 groups of patients with significantly different OS (Table 4). 

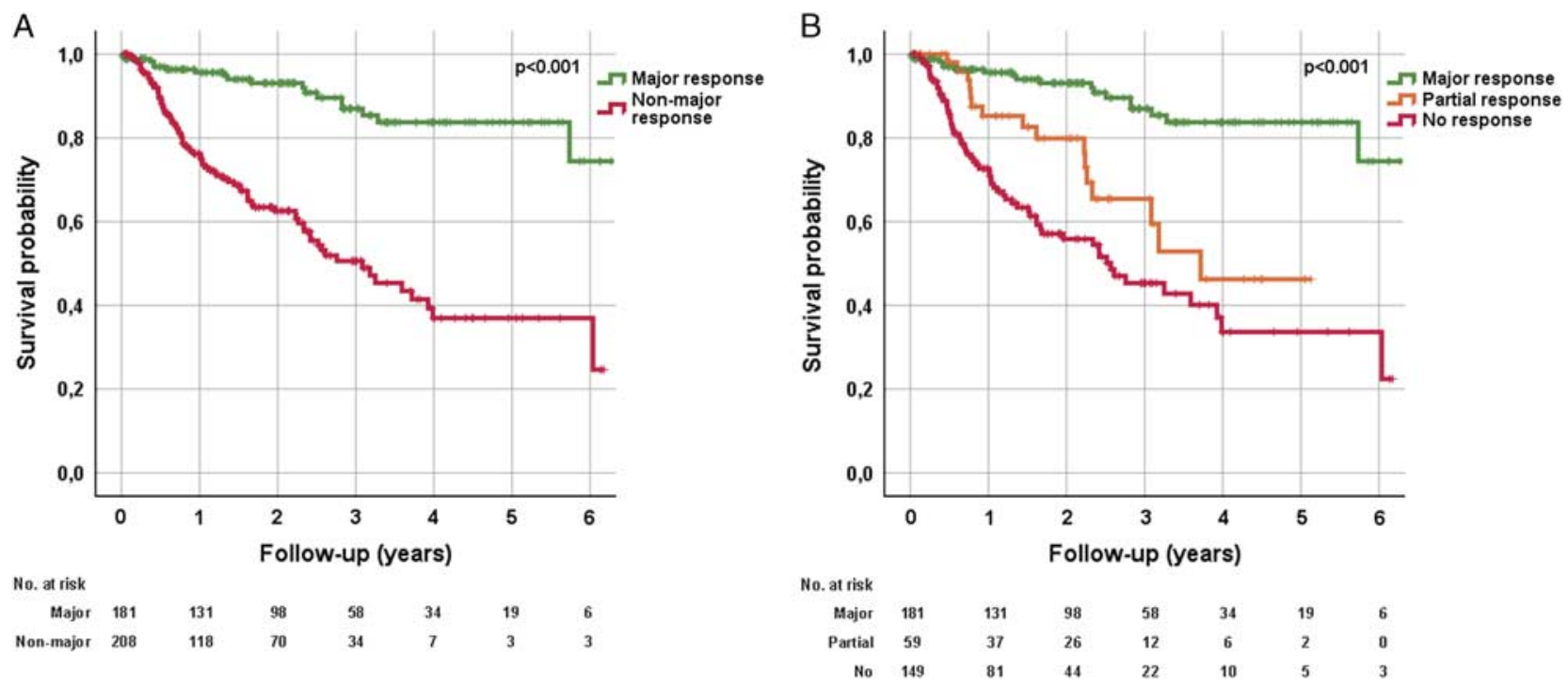

FIGURE 6. Kaplan-Meier plots for OS stratified according to conventional TNM response classification (major responder and nonmajor responder) (A) and TRG integrated with TNM staging (B).

\section{Comparison of Prognostic Models}

To assess the prognostic value of the combination of TRG and TNM in addition to the value of conventional TNM staging, the 2 Cox proportional hazard models (Table 4) were compared using a likelihood ratio test. Model 2 (including the combination of TRG and TNM) had a lower test statistic than model 1 (including conventional response classification) (489.54 vs. $491.64, P=0.041)$. Therefore, the model including TRG and TNM proved to be a more desirable model for prognostication. Moreover, the AIC value was smaller for the combination of TRG and TNM (AIC: 967.58) compared with that for TNM staging alone (AIC: 973.14), indicating that the former has a better prognostic stratification. Sensitivity analysis excluding patients from the center that did not participate in CPR did not impact the study findings (data not shown).

\section{DISCUSSION}

In this retrospective multicenter analysis, we determined histopathologic TRGs in radical cystectomy specimens of muscle-invasive bladder cancer patients after neoadjuvant chemotherapy. TRGs were confirmed by independent pathologic review with low interobserver variability. We could not only validate the prognostic significance of TRG but also provided an easily applicable score, including TNM and TRG, that serves as a prognostic posttreatment classification system. Determination of this score in radical cystectomy specimens after neoadjuvant chemotherapy is simple, reproducible, and provides additional prognostic information. If this can be confirmed in a prospective study, TRG may be routinely added to pathology reports of radical cystectomy specimens after neoadjuvant chemotherapy.

In contrast to other cancers, ${ }^{8-10}$ classification of response to neoadjuvant chemotherapy in muscle-invasive bladder cancer is only based on TNM staging at radical cystectomy. ${ }^{12}$ However, as TNM staging is developed in untreated muscle-invasive bladder cancer, it does not consider specific alterations caused by neoadjuvant chemotherapy and may, therefore, conceal prognostic information in posttreatment specimens. In addition, variability in pathologic TNM staging is a recognized problem in non-muscle-invasive bladder carcinoma, ${ }^{13}$ and some studies suggest that interobserver variability may also affect TNM staging in muscle-invasive bladder cancer. $^{14,15}$ These issues substantiate a need to improve posttreatment stratification.

Fleischmann et $\mathrm{al}^{11}$ were the first to show that histomorphologic classification of postchemotherapy cystectomy specimens into TRGs harbors prognostic information. However, this was a single-center cohort that mainly consisted of patients with advanced muscle-invasive bladder cancer (cT3/4 and/or $\mathrm{cN}+$ ). In the current study, we performed independent validation of the findings of Fleischmann and colleagues in an evenly balanced cohort, including cT2N0 patients. Thus, the present cohort represents a practice of treating "all-comers" with neoadjuvant chemotherapy and confirms that TRG also harbors prognostic information in a clinically lower-staged muscle-invasive bladder cancer cohort.

Our findings may be in contrast to another study investigating the prognostic value of TRG in muscle-invasive bladder cancer. ${ }^{16}$ This multicenter study by Brimo and colleagues also identified TRGs as a prognostic parameter in univariable analyses, but this was not confirmed in multivariable analysis. However, there are some important differences between the study of Brimo and colleagues and our study. First, our sample size met the requirement of the power calculation, while the study of Brimo and colleagues may have been underpowered $(n=165)$. Moreover, we excluded aggressive histomorphologic variants, in line with the large randomized controlled trials on neoadjuvant chemotherapy in 

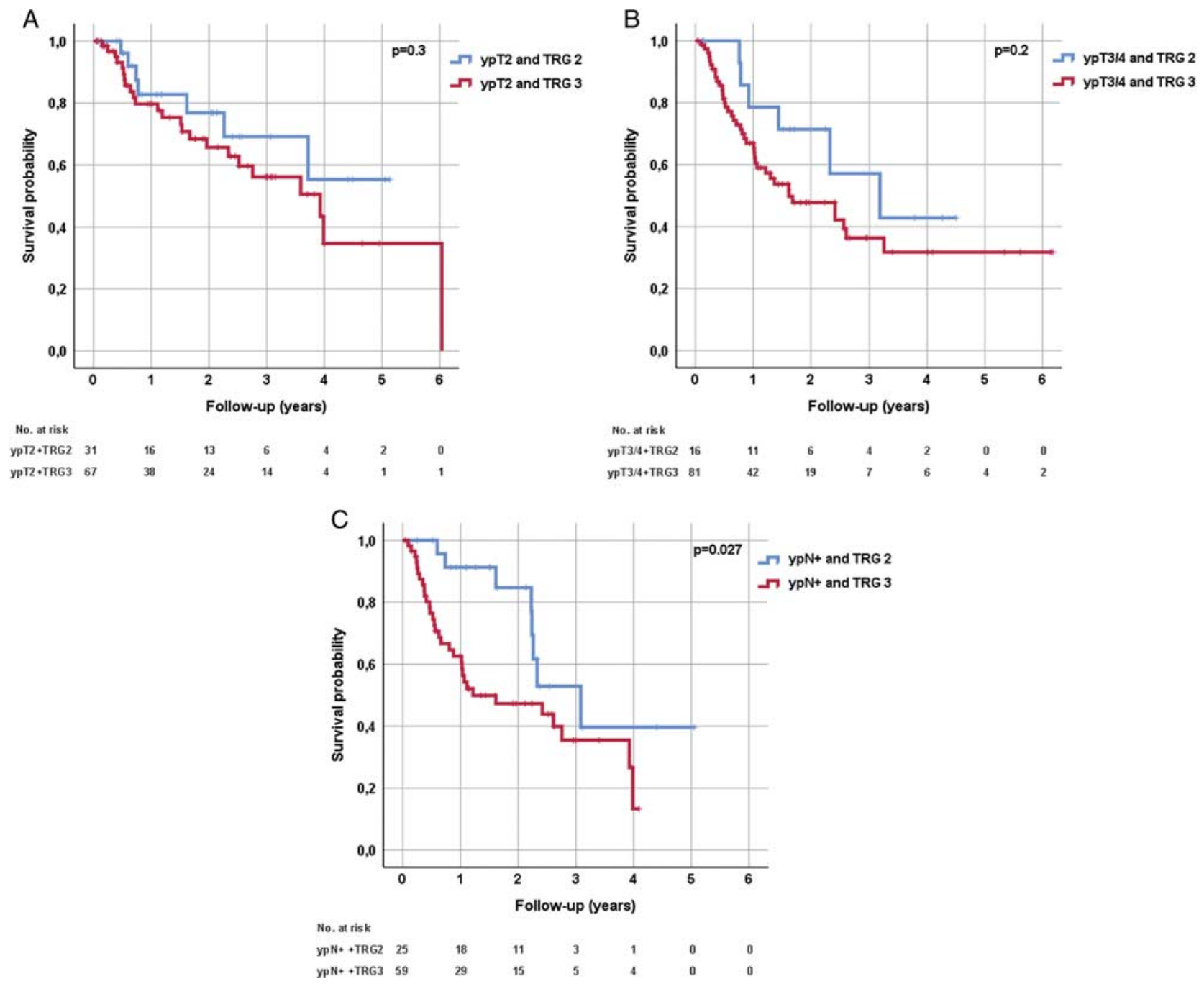

FIGURE 7. Kaplan-Meier plots for OS stratified according to TRG within ypT2 (A), ypT3/4 (B), and ypN1-3 (C).

muscle-invasive bladder cancer, ${ }^{17,18}$ whereas Brimo et al ${ }^{16}$ did not. ${ }^{16}$ Taken together, our study provided a multicenter validation of the prognostic significance of TRG in muscleinvasive bladder cancer after neoadjuvant chemotherapy.

Several reasons may explain why TRGs are commonly used in gastrointestinal malignancies and not in muscle-invasive bladder carcinoma. Histomorphologically, the layers of the gastrointestinal wall are clearly defined, in contrast to the layers of the bladder wall. This may contribute to less accurate clinical staging in bladder carcinoma and a more challenging estimation of tumor shrinkage due to chemotherapy. Moreover, while diagnostic biopsy of, for example, esophageal carcinoma has limited effect on tumor size and architecture, transurethral resection (TUR) of the bladder tumor affects not only tumor size but also histopathologic findings. Two studies addressed the potential confounding effect of TUR in relation to neoadjuvant chemotherapy. Wang et $\mathrm{al}^{19}$ found that both neoadjuvant chemotherapy and TUR cause fibroblastic reaction and necrosis, while hyalinization of the bladder wall is specifically seen after neoadjuvant chemotherapy. Brant et $\mathrm{al}^{20}$ evaluated histologic changes after neoadjuvant chemotherapy in 139 patients with cT2 bladder carcinoma and estimated that $38 \%$ of pathologic response could be attributed to TUR alone. They did not include higher staged patients, and both did not assess clinical outcomes. Moreover, the residual tumor size in relation to the estimated former tumor bed was not considered, although this is an important feature of TRG determination. Even if TUR causes regressive changes, these will be limited to the bladder wall and will neither affect the perivesical fat nor impact the size of the former tumor bed. Finally, the prognostic significance of TRG in the present more evenly balanced cohort including highstage muscle-invasive bladder cancer patients cannot be negated and suggests clinical applicability.

TRG may serve as a useful tool in clinical practice and research for several reasons. First, integrating TRG with TNM staging resulted in more accurate survival prediction and may be helpful in decisions with regard to 
TABLE 3. Univariable Cox Regression Analyses of the Association between Clinicopathologic Factors and OS

\begin{tabular}{|c|c|c|c|}
\hline & HR & $95 \% \mathrm{CI}$ & $\boldsymbol{P}$ \\
\hline Age (continuous) & 0.99 & $0.97-1.01$ & 0.5 \\
\hline \multicolumn{4}{|l|}{ Sex } \\
\hline Male & 1 & & \\
\hline Female & 1.36 & $0.90-2.06$ & 0.14 \\
\hline \multicolumn{4}{|l|}{ NAC regimen } \\
\hline GC & 1 & & \\
\hline MVAC & 1.17 & $0.76-181$ & 0.5 \\
\hline \multicolumn{4}{|l|}{ No. NAC cycles } \\
\hline 3 & 1 & & \\
\hline 4 & 1.09 & $0.71-1.70$ & 0.7 \\
\hline \multicolumn{4}{|l|}{ ypN stage } \\
\hline ypN0 & 1 & & \\
\hline ypN1-3 & 3.34 & $2.21-5.03$ & $<0.001$ \\
\hline \multicolumn{4}{|l|}{ ypT stage } \\
\hline pT0 & 1 & & \\
\hline pT1/a/is & 2.75 & $1.16-6.53$ & 0.022 \\
\hline pT2 & 5.30 & $2.72-10.34$ & $<0.001$ \\
\hline $\mathrm{pT} 3 / \mathrm{T} 4$ & 8.68 & $4.58-16.43$ & $<0.001$ \\
\hline \multicolumn{4}{|l|}{ Histology } \\
\hline Urothelial carcinoma & 1 & & \\
\hline Variant & 1.66 & $0.97-2.84$ & 0.063 \\
\hline \multicolumn{4}{|l|}{ Surgical margin } \\
\hline Negative & 1 & & \\
\hline Positive & 3.36 & $1.74-6.50$ & $<0.001$ \\
\hline \multicolumn{4}{|l|}{ TRG } \\
\hline 1 & 1 & & \\
\hline 2 & 5.81 & $2.77-12.18$ & $<0.001$ \\
\hline 3 & 9.82 & $5.03-19.18$ & $<0.001$ \\
\hline \multicolumn{4}{|l|}{ Conventional response } \\
\hline Major response & 1 & & \\
\hline Nonmajor response & 5.320 & $3.18-8.90$ & $<0.001$ \\
\hline \multicolumn{4}{|c|}{ Response TNM with TRG } \\
\hline Major response & 1 & & \\
\hline Partial response & 3.419 & $1.74-6.73$ & $<0.001$ \\
\hline Nonresponse & 6.169 & $3.65-10.44$ & $<0.001$ \\
\hline
\end{tabular}

Bold value indicates statistically significant $(P<0.05)$.

GC indicates gemcitabine and cisplatin; HR, hazard ratio; MVAC, methotrexate, vinblastine, doxorubicin, and cisplatin; NAC, neoadjuvant chemotherapy.

the timing of follow-up. ${ }^{21}$ Second, TRG could be used to tailor therapeutic adjuvant strategies in patients with residual tumor after neoadjuvant chemotherapy. TRG provides the opportunity to gage antitumor efficacy of agents, and persistence of viable tumor after therapy might indicate the need to alter or explore novel treatments in the adjuvant setting. Third, TRG could be used as a comparative parameter for biomarker studies. A standard approach for grading pathologic response to neoadjuvant chemotherapy is important for the assessment of predictive biomarkers and will facilitate comparison of results across studies.

Our study is limited by its retrospective design, possibly resulting in differences in grossing protocols across institutions. Nevertheless, we performed CPR and found high interobserver agreement. One center could not participate in CPR, but sensitivity analysis restricted to centers who did participate in CPR did not alter the main study findings. Importantly, we enrolled a homogenous cohort of neoadjuvant chemotherapy patients, including only common variant histologies in line with current clinical trials, and all patients received at least 3 cycles of cisplatin-based neoadjuvant chemotherapy.
TABLE 4. Multivariable Cox Regression Analyses of OS

\begin{tabular}{|c|c|c|c|c|c|c|}
\hline \multirow[b]{2}{*}{ Characteristics } & \multicolumn{3}{|c|}{ Model 1} & \multicolumn{3}{|c|}{ Model 2} \\
\hline & HR & $95 \% \mathrm{CI}$ & $\boldsymbol{P}$ & HR & $95 \%$ CI & $\boldsymbol{P}$ \\
\hline Age (continuous) & 1.01 & $0.98-1.02$ & 0.98 & 0.99 & $0.98-1.02$ & 0.92 \\
\hline Female sex & 1.33 & $0.88-2.02$ & 0.18 & 1.27 & $0.83-1.93$ & 0.27 \\
\hline $\begin{array}{l}\text { Positive surgical } \\
\text { margin }\end{array}$ & 1.66 & $0.89-3.10$ & 0.11 & 1.53 & $0.82-2.87$ & 0.19 \\
\hline \multicolumn{7}{|c|}{ Conventional response } \\
\hline Major responder & 1 & & & & & \\
\hline $\begin{array}{l}\text { Nonmajor } \\
\text { responder }\end{array}$ & 5.03 & $2.98-8.49$ & $<0.001$ & & & \\
\hline \multicolumn{7}{|c|}{ Response TNM with TRG } \\
\hline Major responder & & & & 1 & & \\
\hline Partial responder & & & & 3.44 & $1.74-6.81$ & $<0.001$ \\
\hline Nonresponder & & & & 5.75 & $3.36-9.84$ & $<0.001$ \\
\hline
\end{tabular}

Bold value indicates statistically significant $(P<0.05)$.

Model 1 represents conventional response classification, and model 2 represents TRG integrated with TNM staging.

HR indicates hazard ratio.

In conclusion, this study validates TRG as an independent predictor of OS in a multicenter series of patients with muscle-invasive bladder cancer who received cisplatin-based chemotherapy before radical cystectomy. TRG is a simple and highly reproducible measurement of response to neoadjuvant chemotherapy. Importantly, integrating TRG with TNM staging resulted in significantly better prognostic stratification than conventional TNM staging alone. Therefore, we suggest that TRG may routinely be included in postchemotherapy pathologic reports of radical cystectomy specimens. In addition to predicting patient outcome, combining TRG and TNM may be used to tailor postoperative treatment and may serve as a comparative parameter for predictive biomarkers.

\section{REFERENCES}

1. Alfred Witjes J, Lebret T, Compérat EM, et al. Updated 2016 EAU guidelines on muscle-invasive and metastatic bladder cancer. Eur Urol. 2017;71:462-475.

2. Zargar H, Espiritu PN, Fairey AS, et al. Multicenter assessment of neoadjuvant chemotherapy for muscle-invasive bladder cancer. Eur Urol. 2015;67:241-249.

3. Petrelli F, Coinu A, Cabiddu M, et al. Correlation of pathologic complete response with survival after neoadjuvant chemotherapy in bladder cancer treated with cystectomy: a meta-analysis. Eur Urol. 2014;65:350-357.

4. Zargar H, Zargar-Shoshtari K, Lotan Y, et al. Final Pathological stage after neoadjuvant chemotherapy and radical cystectomy for bladder cancer-does pT0 predict better survival than $\mathrm{pTa} / \mathrm{Tis} / \mathrm{T} 1$ ? J Urol. 2016;195:886-893.

5. Van Allen EM, Mouw KW, Kim P, et al. Somatic ERCC2 mutations correlate with cisplatin sensitivity in muscle-invasive urothelial carcinoma. Cancer Discov. 2014;4:1140-1153.

6. Plimack ER, Dunbrack RL, Brennan TA, et al. Defects in DNA repair genes predict response to neoadjuvant cisplatin-based chemotherapy in muscle-invasive bladder cancer. Eur Urol. 2015;68:959-967.

7. Groenendijk FH, De Jong J, Fransen Van De Putte EE, et al. ERBB2 mutations characterize a subgroup of muscle-invasive bladder cancers with excellent response to neoadjuvant chemotherapy. Eur Urol. 2016;69:384-388.

8. Mandard AM, Dalibard F, Mandard JC, et al. Pathologic assessment of tumor regression after preoperative chemoradiotherapy of esophageal carcinoma. Clinicopathologic correlations. Cancer. 1994; 73:2680-2686. 
9. Mancini R, Pattaro G, Diodoro MG, et al. Tumor regression grade after neoadjuvant chemoradiation and surgery for low rectal cancer evaluated by multiple correspondence analysis: ten years as minimum follow-up. Clin Colorectal Cancer. 2018;17:e13-e19.

10. Langer R, Becker K, Zlobec I, et al. A multifactorial histopathologic score for the prediction of prognosis of resected esophageal adenocarcinomas after neoadjuvant chemotherapy. Ann Surg Oncol. 2014;21:915-921.

11. Fleischmann A, Thalmann GN, Perren A, et al. Tumor regression grade of urothelial bladder cancer after neoadjuvant chemotherapy: a novel and successful strategy to predict survival. Am J Surg Pathol. 2014;38:325-332.

12. Sobin H, Gospodarowicz MK, Wittekind C. TNM Classification of Malignant Tumours, 7th edition. New York: Wiley-Lyss Inc; 2009.

13. Van Rhijn BWG, Van Der Kwast TH, Kakiashvili DM, et al. Pathological stage review is indicated in primary pT1 bladder cancer. BJU Int. 2009;106:206-211.

14. Ananthanarayanan V, Pan Y, Tretiakova M, et al. Influence of histologic criteria and confounding factors in staging equivocal cases for microscopic perivesical tissue invasion (pT3a). Am J Surg Pathol. 2014;38:167-175.

15. Paner GP, Montironi R, Amin MB. Challenges in pathologic staging of bladder cancer: proposals for fresh approaches of assessing pathologic stage in light of recent studies and observations pertaining to bladder histoanatomic variances. Adv Anat Pathol. 2017;24:113-127.

16. Brimo F, Downes MR, Jamaspishvili T, et al. Prognostic pathological factors in radical cystectomy after neoadjuvant chemotherapy. Histopathology. 2018;73:732-740.

17. Grossman HB, Natale RB, Tangen CM, et al. Neoadjuvant chemotherapy plus cystectomy compared with cystectomy alone for locally advanced bladder cancer. $N$ Engl J Med. 2003;349:859-866.

18. Advanced Bladder Cancer (ABC) Meta-analysis Collaboration. Neoadjuvant chemotherapy in invasive bladder cancer: Update of a systematic review and meta-analysis of individual patient data. Eur Urol. 2005;48:202-205.

19. Wang HJ, Solanki S, Traboulsi S, et al. Neoadjuvant chemotherapyrelated histologic changes in radical cystectomy: assessment accuracy and prediction of response. Hum Pathol. 2016;53:35-40.

20. Brant A, Kates M, Chappidi MR, et al. Pathologic response in patients receiving neoadjuvant chemotherapy for muscle-invasive bladder cancer: is therapeutic effect owing to chemotherapy or TURBT? Urol Oncol. 2017;35:34.e17-34.e25.

21. Zuiverloon TCM, van Kessel KEM, Bivalacqua TJ, et al. Recommendations for follow-up of muscle-invasive bladder cancer patients: a consensus by the international bladder cancer network. Urol Oncol. 2018;36:423-431. 